\title{
$5 \quad$ Ergebnisse zur Akzeptanz des mehrsprachigen Vorlesens
}

\author{
Robert Hilbe, Sabine Kutzelmann, Ute Massler und Klaus Peter
}

Im Vergleich zu den im vorausgehenden Kapitel dargestellten formativen Ergebnissen, die der schrittweisen Optimierung der einzelne Designkomponenten dienten, wird nun eine holistische Sicht auf das voll entwickelte Unterrichtsdesign Mehrsprachiges Vorlesen durch die Lehrperson (MeVoL) und seine Potenziale angestrebt. Gemäß Nieveen/ Folmer (2013: 155) entspricht dies der summativen Evaluationsphase als Abschluss einer Design-Based-Research-Studie. Eine solche summative Überprüfung ist erst dann sinnvoll, wenn die Intervention soweit ausgereift ist, dass eine potenzielle Effektivität gegeben ist. Dafür muss das entwickelte Design überzeugend sein, d.h. relevant für die Problemstellung, logisch konzipiert und praktisch umsetzbar. Die Ausführungen in den vorausgehenden Abschnitten haben gezeigt, dass diese Bedingungen durch die wiederholte Erprobung in den Projektklassen, die Evaluation und die mehrfache zyklische Überarbeitung des Unterrichtsdesigns weitgehend erfüllt sind. Unser Forschungsinteresse in der Phase des Projektabschlusses galt daher der Akzeptanz des mehrsprachigen Unterrichtsdesigns MeVoL durch Lehrpersonen und Schüler/innen, da darin notwendige Voraussetzungen für gelingende Lernprozesse und die weitere Nutzung von MeVoL nach Projektende gesehen werden (vgl. 2.2). Die folgenden Ergebnisse stellen die Akzeptanz aus der Perspektive der Schüler/innen und der Lehrpersonen dar: Wie nehmen die Schüler/innen MeVoL im Vergleich zu ihrem bisherigen Fremdsprachenunterricht wahr? Wie schätzen die Lehrpersonen den Nutzen, den Aufwand und die Rahmenbedingungen für den Einsatz von MeVoL ein? Zur Beantwortung dieser und weiterer Fragen werden Daten aus den Feedbackbogen von Lehrpersonen und Schüler/innen aus Zyklus 7 verwendet. Sie werden mit qualitativen Statements aus den Interviews illustriert, die nach Zyklus 6 durchgeführt wurden. Die Darstellung der Ergebnisse stützt sich auf die theoretischen Überlegungen zur Akzeptanz in Abschnitt 2.2, der interessierten Leser/innen zur vorgängigen Lektüre empfohlen wird.

Der erste Teil dieses Kapitels widmet sich der Akzeptanz von MeVoL aus Schülersicht (5.1). Es wird ausgeführt, wie gut MeVoL den Schüler/innen gefallen hat, was die Gründe dafür sind und wie sie die Nützlichkeit der einzelnen Design-Komponenten einschätzen. MeVoL wird aus Schülersicht mit dem bisherigen Unterricht und anderen (Vor-)Leseformen verglichen. Schließlich werden Differenzen in der Akzeptanz bei den Lernenden in Abhängigkeit von der verwendeten Fremdsprache (Englisch vs. Französisch) dargestellt. Im zweiten Teil werden die Ergebnisse zur Akzeptanz aus Lehrersicht präsentiert (5.2). Sie geben Antwort auf die Frage, wie nützlich die Lehrpersonen MeVoL einschätzen, welcher Aufwand für sie mit der Verwendung von MeVoL verbun- 
den war und ob die Lehrpersonen MeVoL weiterhin einsetzen möchten. Die Ergebnisse beider Perspektiven werden dann synoptisch zusammengefasst (5.3). Abschließend werden jene Bereiche angesprochen, in denen die Akzeptanz des Unterrichtsdesigns kritisch beurteilt werden muss, es wird nach möglichen Erklärungen gesucht, und es werden Konsequenzen diskutiert, die für die Weiterentwicklung des Designs genutzt werden können.

\subsection{MeVoL aus Schülersicht}

\subsubsection{Hat den Schüler/innen das mehrsprachige Vorlesen gefallen?}

MeVoL hat einem Großteil der befragten Schüler/innen (84.4\%) insgesamt gut bis sehr gut gefallen. Die meiste Zustimmung erhält dabei das Statement „MeVoL war eine gute Abwechslung zum sonstigen Fremdsprachenunterricht" (83.5\%, M=3.2, SD=.79, $\mathrm{N}=188$ ). Auch die Begründung, dass das mehrsprachige Vorlesen gemocht wird, weil man beim Zuhören genießen kann und nicht selber lesen muss, wird stark befürwortet (79.1\%, M=3.1, SD=.89, N=186). Das Vorgelesen-Bekommen wird im Vergleich zum Selber-Lesen von vielen Lernenden als sinnvolle kognitive Entlastung erlebt, wie folgende Aussage eines Schülers im Interview ${ }^{1}$ zeigt:

I: Du hast jetzt gesagt, ist noch cool, wenn man so etwas vorgelesen bekommt. Ist das [...] cooler als Selber-Lesen, Vorgelesen-Bekommen?

B: Mhmm, also ich finde schon, weil dann kann man es sich wie, ja eben besser vorstellen, und ... und ja dann kann man, ist man irgendwie wie entspannter, wenn man dann zuhören kann.

I: Mhmm. Ist Zuhören weniger anstrengend als Selber-Lesen?

B: Ja. (Interview S ANDIVIo7 CH Z6: 186-189)

Ein weiterer, ebenso zustimmend beantworteter Grund dafür, dass MeVoL vielen Lernenden gefällt, liegt darin, dass sie gerne Geschichten hören (75.1\% zustimmende Antworten, $\mathrm{M}=3.0, \mathrm{SD}=.83, \mathrm{~N}=185$ ).

Deutlich weniger hat den Lernenden die Anschlusskommunikation Spaß gemacht: Nur etwas mehr als der Hälfte (51.9\%, M=2.5, SD=.92 N=185) hat gefallen, dass sie mit anderen darüber sprechen konnte, was sie an der Geschichte gut oder schlecht fand. Noch etwas weniger Schüler/innen (49.2\%, M=2.49, SD=.93, N=187) fanden es interessant, sich mit Mitschüler/innen über den Text auszutauschen. Offensichtlich stellt das Gelingen einer interessanten Anschlusskommunikation, die die Lernenden anspricht,

1 Zitate von Schüler/innen und Lehrpersonen wurden im gesamten Handbuch zugunsten einer besseren Lesbarkeit sprachlich geglättet. 
eine Herausforderung bei der Umsetzung von MeVoL dar. Das Herstellen eines Klassengesprächs, das einen Großteil der Lernenden involviert, scheint in einigen Fällen nicht geglückt zu sein:

I: Wie waren für dich diese Gespräche, so die Gespräche nach dem Vorlesen, wie fandest du die?

B: Ich war eigentlich der Einzige, der mitmachte.

I: Wirklich? Erzähle mal!

B: Also sie haben auch ein paar Mal gesagt: Ist es jetzt echt nur er, der es weiß, weil ich mich gemeldet hatte, dabei haben es auch andere gewusst, aber nur wirklich ich habe mich gemeldet. (Interview S DAERBEo6 CH Z6: 264-267)

Grund dafür könnte u.a. sein, dass die Lernenden es nicht gewohnt sind, bewertend über Texte zu sprechen. Anschlusskommunikation gehört gewissermaßen nicht zu ihren vertrauten schulischen Handlungsroutinen (vgl. dazu die Ausführungen zur Praktikanz in 2.2.1). Der Nutzen der Anschlusskommunikation ist für sie nicht klar, und das Sprechen über das Vorgelesene führt daher zu Irritation:

I: Ihr habt nach dem Vorlesen zwei-, dreimal darüber gesprochen, wie ihr den Text selbst findet oder welche Passagen euch gut oder nicht gut gefallen haben. Oder ihr habt darüber gesprochen, was der Erzähler jetzt noch machen könnte. Diese Gespräche, wie fandest du die?

B: Einerseits verwirrend, andererseits auch ein bisschen sinnlos, also ... ich weiß nicht, wieso man das unbedingt jetzt gerade fragen muss, ob es einem gefiel oder nicht. (Interview S PERFWI12 CH Z6: 278-79)

\subsubsection{Wie beurteilen die Lernenden die Nützlichkeit der einzelnen Design-Komponenten?}

Die Lernenden wurden danach gefragt, wie nützlich folgende Gestaltungsmerkmale des Unterrichtsdesigns für ihr Textverständnis waren: die verwendeten deutschen Vorleseteile, die verwendeten Bilder, die Aufgaben vor dem Vorlesen (pre-scaffolding), die Aufgaben nach dem Vorlesen zur Verständnissicherung (post-scaffolding), das Sprechen über die Geschichte mit der Lehrperson oder mit Mitschüler/innen und die Beschäftigung mit Hörstrategien.

Abbildung 1 stellt die wahrgenommene Nützlichkeit dieser Komponenten aus Schülersicht nach absteigendem Zustimmungsgrad dar. Die größte Unterstützung für das Verständnis sahen die Lernenden in den deutschsprachigen Vorleseteilen $(82.3 \%$ zustimmende Antworten, $\mathrm{M}=3.3, \mathrm{SD}=.82, \mathrm{~N}=187$ ), gefolgt vom Sprechen über die Geschichte mit der Lehrperson oder mit Mitschüler/innen (75.6\%, M=3.0, SD=.82, N=185) und der Verwendung der Bilder (71.8\%, M=2.9, SD=.94, N=188). Die Beschäftigung mit Hörstrategien $(60.2 \%, \mathrm{M}=2.6, \mathrm{SD}=.84, \mathrm{~N}=186)$ sowie das Beantworten von Fragen und 
das Lösen von Aufgaben nach dem Vorlesen (50.8\%, M=2.6, SD=.91, N=187) wurden demgegenüber als weniger nützlich für das Verständnis eingestuft. In der Abbildung nicht dargestellt, jedoch zusätzlich interessant ist, dass nur rund ein Viertel der Schüler/innen den Sprachwechsel als verwirrend empfunden hat $(25.2 \%, \mathrm{M}=1.9, \mathrm{SD}=.88$, $\mathrm{N}=187$ ). Auch in den qualitativen Schülerinterviews überwog die positive Wahrnehmung der Kombination von Schul- und Fremdsprache (22 positive Codings gegenüber 4 negativen und 9 neutralen, $\mathrm{N}=18$ ). Insgesamt wird damit das mehrsprachige Design von einem Großteil der Schüler/innen als sinnvoll und hilfreich wahrgenommen, wie folgende Schüleraussage illustriert: „Also das mehrsprachige Vorlesen finde ich gut, weil wenn man das Englische nicht wirklich versteht, hat man immer noch das Deutsche [...]" (Interview S PEUTRA01 D Z6: 57).

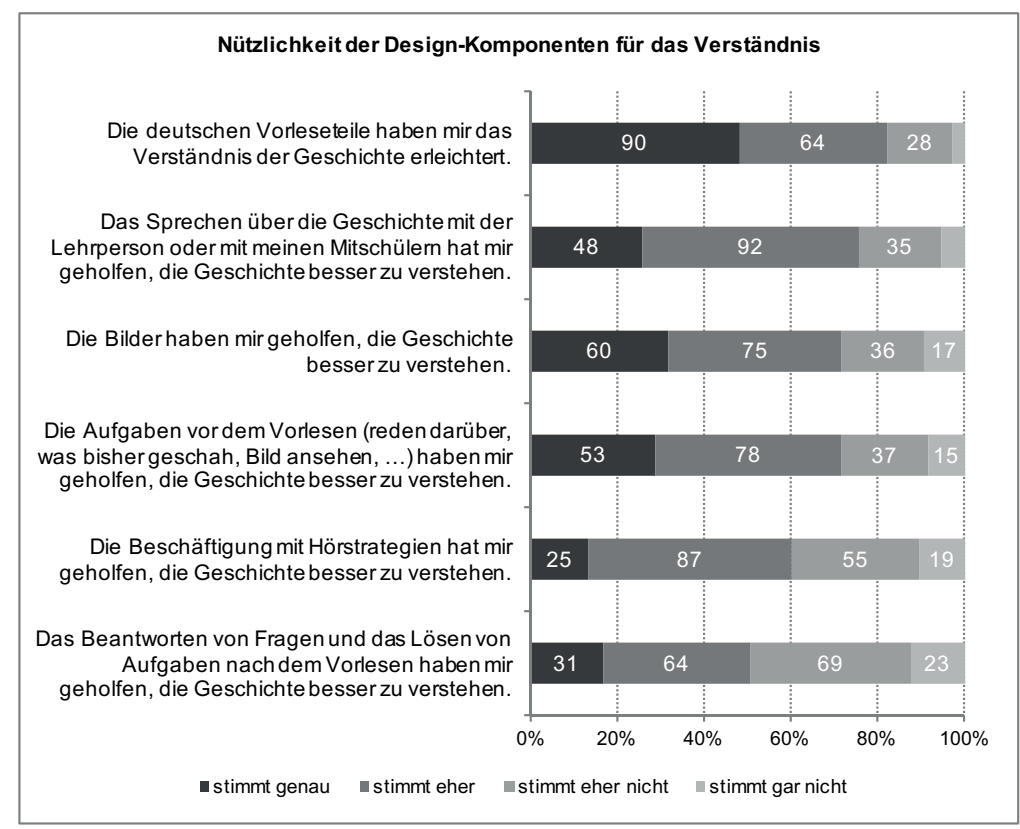

Abbildung 1: Nützlichkeit der Design-Komponenten aus Schülersicht $(\mathrm{N}=187$, absolute Häufigkeiten nach absteigender Zustimmung)

\subsubsection{Wie nehmen die Schüler/innen das mehrsprachige Vorlesen im Vergleich zum regulären Fremdsprachenunterricht wahr?}

Die Schüler/innen wurden gebeten, MeVoL mit ihrem bisherigen Fremdsprachenunterricht bei derselben Lehrperson zu vergleichen. Es wurde dazu ein Polaritätenprofil mit beschreibenden Adjektiven auf einer Skala von 1 bis 7 eingesetzt, wobei dem Wert 4 kein Unterschied zum bisherigen Unterricht entspricht (vgl. Abb. 2). Die Ergebnisse zei- 
gen, dass MeVoL sich hauptsächlich in folgenden Aspekten vom bisherigen Unterricht abhebt:

1. geringerer erlebter Aufwand (cost): weniger anstrengend ( $M=2.8, S D=1.29, N=188)$, entspannter $(M=5.3, S D=1.60, N=186)$, geringerer Zeitdruck $(M=3.1, S D=1.31$, $\mathrm{N}=187)$, jedoch dennoch verständlicher $(\mathrm{M}=4.6, \mathrm{SD}=1.45, \mathrm{~N}=188)$

2. bessere Emotion und Motivation (intrinsic value): interessanter $(\mathrm{M}=4.7, \mathrm{SD}=1.79$, $\mathrm{N}=188)$, abwechslungsreicher $(\mathrm{M}=4.6, \mathrm{SD}=1.64, \mathrm{~N}=188)$, bessere Stimmung $(\mathrm{M}=4.8$, $\mathrm{SD}=1.62$, N=187), höhere Konzentration ( $\mathrm{M}=4.4, \mathrm{SD}=1.50, \mathrm{~N}=184$ )

3. bessere Organisation des Unterrichts: bessere Organisation $(\mathrm{M}=4.7, \mathrm{SD}=1.41, \mathrm{~N}=187)$, klarere Aufgabenstellung ( $M=4.6, \mathrm{SD}=1.40, \mathrm{~N}=188)$

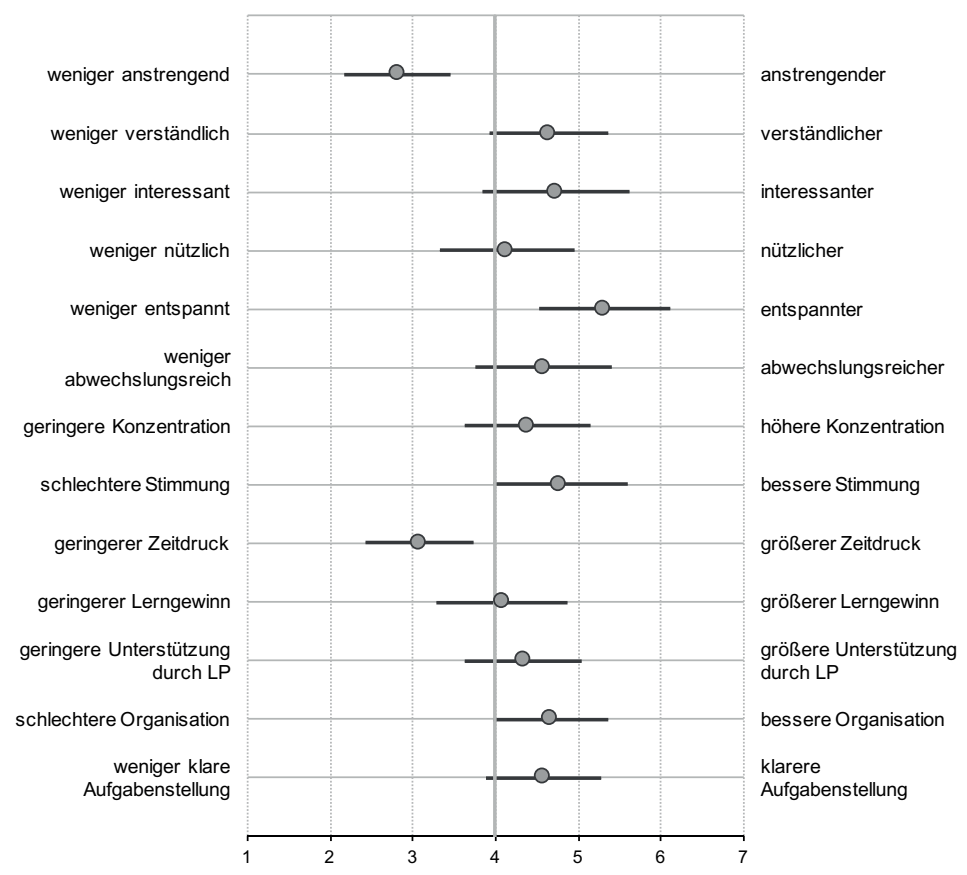

Abbildung 2: Wahrnehmung von MeVoL aus Schülersicht - Polaritätenprofil im Vergleich zum bisherigen Unterricht ( $\mathrm{N}=188$, Mittelwerte und Standardabweichungen)

In Bezug auf den wahrgenommenen Nutzen (utility value) zeigen sich aus Schülersicht hingegen keine deutlichen Differenzen zum bisherigen Unterricht (Nützlichkeit: $M=4.1$, $\mathrm{SD}=1.62, \mathrm{~N}=188$; Lerngewinn: $\mathrm{M}=4.1, \mathrm{SD}=1.58, \mathrm{~N}=187$ ). Dies zeigt sich auch an einem weiteren Item, das nicht Teil des Polaritätenprofils ist: Die Schüler/innen wurden danach gefragt, wie viel ihnen MeVoL für das Lernen im Fremdsprachenunterricht gebracht habe. Die Einschätzung fällt eher moderat aus (59.3\% zustimmende Antworten, $\mathrm{M}=2.6, \mathrm{SD}=.86, \mathrm{~N}=187$ ). 
Zusammenfassend kann festgestellt werden, dass die Akzeptanz von MeVoL als neues - und unter Umständen fremdes - Element des Unterrichts bei den Lernenden insgesamt vorhanden ist. Im Vergleich zum bisherigen Unterricht werden vor allem der erlebte Aufwand sowie der affektive und motivationale Anteil (Emotion und Interesse) deutlich positiver wahrgenommen. Der kognitive Anteil, d.h. der wahrgenommene Nutzen wird vergleichbar mit dem regulären Fremdsprachenunterricht eingestuft. Dies ergibt insgesamt eine positive Bilanz für die Wahrnehmung von MeVoL: Trotz des gefühlten geringeren Aufwands ist der wahrgenommene Nutzen identisch mit dem bisherigen Unterricht. Zentral ist aus unserer Sicht jedoch der positive Effekt von MeVoL auf die Emotionen und die Motivation der Lernenden, da damit wichtige psychologische Voraussetzungen für gelingende Lernprozesse geschaffen werden (vgl. 3.1.2).

Es muss jedoch berücksichtigt werden, dass ein Neuigkeitseffekt sehr wahrscheinlich ist, der die Einschätzung von MeVoL positiv beeinflussen dürfte. So zeigen auch die häufigen offenen Antworten im Schülerfeedbackbogen (23 von 149 positiven Nennungen auf der Kategorie Abwechslung vom Unterricht, vgl. 5.1.5) und die Antworten in den Schülerinterviews, dass MeVoL vorwiegend deshalb geschätzt wurde, weil es eine Abwechslung zum regulären Fremdsprachenunterricht, der den Fokus wahrscheinlich stärker auf Schriftlichkeit und Grammatik legt, darstellt: „[...] Es war gut, also es war auch mal etwas anderes als sonst immer da im Buch Aufgaben zu lösen oder so" (Interview S LEERDI05 CH Z6: 149).

\subsubsection{Wie beurteilen die Schüler/innen das mehrsprachige Vorlesen im Vergleich zu alternativen (Vor-)Leseaktivitäten im Fremdsprachenunterricht?}

Wir haben die Schüler/innen im Feedbackbogen gefragt, auf welche Art sie sich gerne mit fremdsprachigen Texten auseinandersetzen: leise selber lesen, laut vorlesen in der Klasse, Geschichten auf CD anhören sowie fremdsprachige Geschichten vorgelesen bekommen (vgl. Abb. 3). Die Akzeptanz des mehrsprachigen Vorlesens schneidet im Vergleich zu allen anderen Varianten deutlich am positivsten ab $(74.2 \%$ zustimmende Antworten, $\mathrm{M}=3.1, \mathrm{SD}=.98, \mathrm{~N}=163$ ). Das mehrsprachige Vorlesen übertrifft in seiner Beliebtheit auch das Vorlesen von ausschließlich fremdsprachigen Geschichten. Der mehrsprachigkeitsdidaktische Ansatz besitzt folglich aus Sicht der Schüler/innen einen Mehrwert im Vergleich zu einem monolingualen Zugang. 


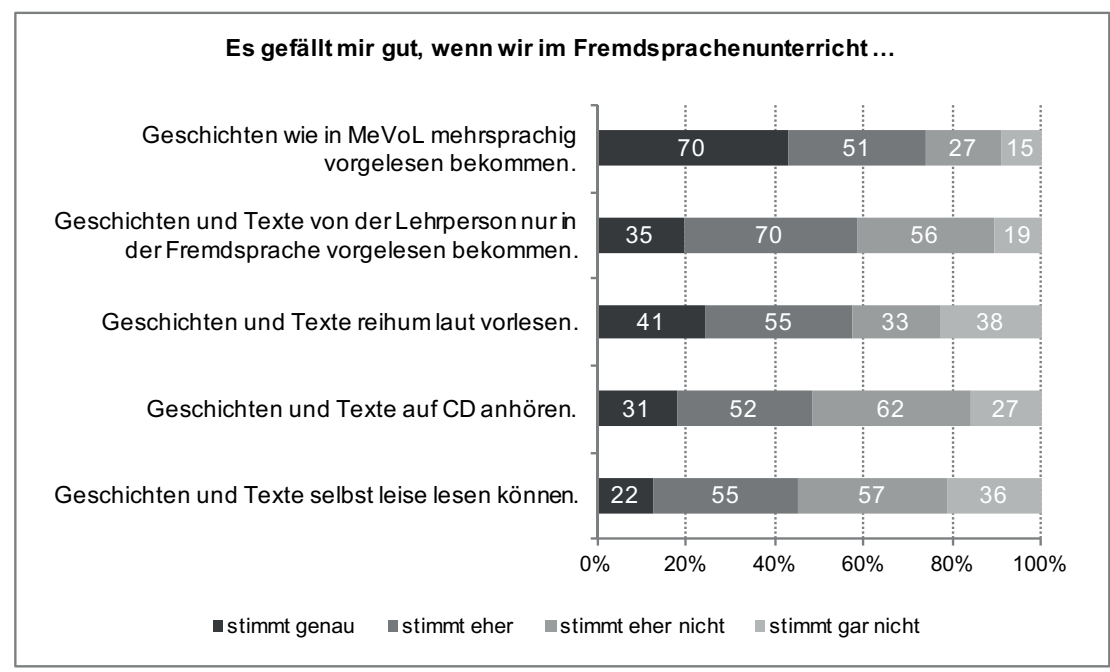

Abbildung 3: MeVoL im Vergleich zu anderen (Vor-)Leseaktivitäten im Fremdsprachenunterricht aus Schülersicht ( $\mathrm{N}=180$, absolute Häufigkeiten nach absteigender Zustimmung)

\subsubsection{Wie begründen Schüler/innen ihre Akzeptanz von MeVoL?}

Die Schüler/innen wurden im Feedbackbogen gefragt, ob ihnen das mehrsprachige Vorlesen gefallen hat und ob sie daher nochmals eine mehrsprachige Geschichte hören möchten. 149 von insgesamt 184 Personen (72\% der Antworten) antworteten mit Ja und gaben einen entsprechenden Grund an. 47 Personen (22\% der Antworten) antworteten mit Nein und gaben Begründungen dafür an, keine mehrsprachige Geschichte mehr hören zu wollen. ${ }^{2}$ Tabelle 1 stellt die kategorisierten Begründungen für die zustimmenden und ablehnenden Antworten dar.

2184 Personen haben auf die Frage geantwortet. Zwölf dieser Personen (6\% der Antworten) haben beide Antwortmöglichkeiten angekreuzt. Insgesamt wurden also 196 Antworten gegeben. Oft wurden für die Antworten zudem zwei oder mehr Begründungen angegeben. Teilaussagen einer Antwort wurden in der folgenden Auswertung verschiedenen Kategorien zugeordnet. Die Gesamtsumme aller Kategorieneinträge übersteigt daher die Anzahl der offenen Antworten. 
Tabelle 1: Begründungen zur Akzeptanz bzw. Ablehnung von MeVoL aus Schülersicht (Anzahl Nennungen)

\begin{tabular}{|l|l|}
\hline $\begin{array}{l}\text { Ja, ich möchte nochmals eine mehrsprachige } \\
\text { Geschichte vorgelesen bekommen. }\end{array}$ & $\begin{array}{l}\text { Nein, ich möchte keine mehrsprachige } \\
\text { Geschichte mehr vorgelesen bekommen. }\end{array}$ \\
\hline $\begin{array}{l}\text { - positive Bewertung des fremdsprachlichen } \\
\text { Lerngewinns (40) }\end{array}$ & - Langeweile (23) \\
- Spaß (33) & - negative Bewertung der Texte (11) \\
- positive Bewertung der Texte (32) & - - negative Bewertung des Lerngewinns (8) \\
- Interesse (25) & - Kritik am MeVoL-Design (4) \\
- Abwechslung vom Unterricht (23) & \\
- Kompetenzerleben (18) & \\
- Gefallen (18) & \\
- Abschalten/Entspannen (15) & - besser bzw. kurzweiliger als normaler Unter- \\
richt (9) & \\
- kein Unterricht (8) & \\
\hline
\end{tabular}

In den nächsten Abschnitten werden die Kategorien erläutert und mit Beispielen offener Antworten aus dem Feedbackbogen aus Zyklus 7 sowie mit Belegstellen aus den Schülerinterviews aus Zyklus 6 illustriert.

Begründungen zur Akzeptanz: Am häufigsten begründeten die Schüler/innen ihre Aussage, weshalb sie nochmals eine mehrsprachige Geschichte vorgelesen bekommen möchten, damit, dass sich MeVoL positiv auf das fremdsprachliche Lernen allgemein bzw. auf das Erlernen weiterer Sprachen auswirke. 22 dieser Begründungen waren zumeist recht unspezifischer Natur und gaben wenige oder keine konkreten Begründungen, weshalb die Schüler/innen dieser Ansicht waren oder in welchen Bereichen des Lernens sie positive Effekte sahen. Aussagen wie „[...] es hilft mir auch viel in Englisch und generell [in den] Fremdsprachen besser zu werden" (Feedbackbogen S SWEFRA09 D Z7) waren hierfür typisch. Weitere Aussagen spezifizierten Lerneffekte im Bereich des Wortschatzes (6 Nennungen) sowie der Aussprache (3 Nennungen). Darüber hinaus sahen zwölf Schüler/innen den Mehrwert von MeVoL im Strategielernen und dessen positiven Effekten für das Verstehen fremdsprachlicher Texte. Ein/e Schüler/in meinte, „[...] ich lerne Strategien anzuwenden und so englische Texte oder Geschichten besser verstehen" (Feedbackbogen S RECKRA03 D Z7). Ein/e andere/r Schüler/in wiederum sah Vorteile für die Konzentrationsfähigkeit (Interview S SLJERA08 D Z6: 87-88).

Die Vermutung liegt nahe, dass die positive Einschätzung des fremdsprachlichen Lernertrags auch darin begründet war, dass sich die Lernenden häufig als kompetente Fremdsprachenlernende während der MeVoL-Unterrichtseinheiten erlebten, da sie der Geschichte gut folgen konnten. 18 Lernende gaben an, die Geschichten gut bis sehr gut zu verstehen, wobei drei davon die Kombination von Schul- und Fremdsprache im MeVoL-Design oder den Einsatz von Mimik und Gestik durch die Lehrperson beim Vorlesen (1 Nennung) als ursächlich hierfür erachteten: „Ja, wie gesagt, dass wir halt auf Deutsch vorgelesen bekommen, das hilft mir halt, dass ich es besser versteh und besser in die Geschichte reinkomme, ja, das hilft mir halt so das Deutsche" (Interview $\mathrm{S}$ ZIMWE03 D Z6: 36). 
Zwei Lernende gaben an, von der Strategievermittlung und -anleitung profitiert zu haben, und sie achteten als Folge davon bewusst darauf, sich nicht ablenken zu lassen:

I: Und warum, woran hast du es nochmal festgemacht, dass du es besser verstanden hast, was hast du gesagt, ist wahrscheinlich der Hauptgrund?

B: Weil ich, so lange wir gelesen haben, auch überlegt habe und mich nicht ablenken ließ. Und dann danach, als wir besprochen haben, was war, dass ich es meistens richtig hatte. (Interview S CLNSRAo6 D Z6: 135-142)

Positiv ist ebenfalls zu vermerken, dass Lernende meinten, der Geschichte folgen zu können, obwohl sie sprachlich nicht alles verstanden hatten und somit eine Frustrationstoleranz zeigten, die essenziell für jede fremdsprachliche Kommunikation ist:

I: Also hast du das eher gut verstanden oder eher nicht so gut verstanden, was man euch da vorgelesen hat?

B: Das meiste habe ich gut verstanden, aber dann waren halt noch manche [...] Sätze oder Wörter, die ich nicht verstanden habe, aber im Großen und Ganzen habe ich's eigentlich gut verstanden. (Interview S CAANHOo6 Ö Z6: 226-231)

Die Kategorien „Spaß“ (insgesamt 33 Nennungen, davon 10 Nennungen „Spaß am Zuhören/Vorlesen“), „Interesse“ (25 Nennungen), „Gefallen“ (18 Nennungen) und „Abschalten/Entspannen“ (15 Nennungen) liegen inhaltlich nahe beieinander und beziehen sich auf den von den Lernenden wahrgenommenen tätigkeitsbezogenen bzw. intrinsischen Wert des Vorlesens (vgl. 2.2.3). Die starke Ausprägung dieses Kategorienkonglomerats lässt vermuten, dass die Akzeptanz von MeVoL vor allem auf das Interesse und das Gefallen der Schüler/innen am Vorlesen mehrsprachiger Texte zurückzuführen ist. Die folgende Aussage illustriert, wie Lernende den Faktor „Entspannung“ damit verknüpften: „[...] es immer sehr interessant ist und auch Spaß macht. Man kann es sich wohl machen und schön zuhören“ (Feedbackbogen S YEARD009 Ö Z7). Sämtliche soeben genannten Kategorien waren thematisch eng mit Äußerungen zur „positiven Bewertung der Texte“ (32 Nennungen) verbunden bzw. wurden oft gemeinsam angeführt. Dies zeigt die Bedeutung, die der Eignung der Vorlesetexte als dem zentralen, interessestiftenden Inhalt der MeVoL-Unterrichtseinheiten zukam. Gefielen den Lernenden die Texte, so begrüßten sie weitere Vorlesegeschichten mit Begründungen wie „weil es cool ist“ (Feedbackbogen S PINOFE07 Ö Z7) oder „es ist sehr interessant und spannend. Es gefällt mir sehr und ich liebe es, wenn wir über Geschichten reden“ (Feedbackbogen S SAANH007 Ö Z7).

Die Akzeptanz von MeVoL wurde auch in den offenen Fragen häufig mit der Abwechslung zum regulären Unterricht (23 Nennungen) begründet (vgl. hierzu die Ausführungen unter 5.1.3 zu den geschlossenen Fragen), wobei weitere neun Schüler/innen MeVoL selbst besser als ihren normalen Unterricht beurteilten. Es muss jedoch auch erwähnt werden, dass insgesamt achtmal weitere Vorlesegeschichten gewünscht wurden, weil sie „Unterricht wegnehmen“ bzw. als „kein Unterricht“ erlebt wurden. 
Begründungen zur Ablehnung: Das am häufigsten genannte ablehnende Argument war die Langeweile (23 Nennungen), die MeVoL bei den Lernenden auslöste, „da es immer das Gleiche ist und langweilig wird" (Feedbackbogen S TANOBA09 D Z7). Langeweile mag auch durch mangelndes Interesse an den Texten bzw. deren negative Einschätzung (11 Nennungen) ausgelöst werden, wie folgende offene Antwort verdeutlicht: „[...] weil die Texte immer langweilig sind. Und mich nicht interessieren“ (Feedbackbogen S SUERRA11 D Z7).

Neun Schüler/innen nannten Verständnisschwierigkeiten als Grund, weshalb sie keine mehrsprachigen Texte mehr hören möchten. Diese Begründung schien insbesondere für Schweizer Lernende relevant zu sein, auf die sechs der neun Aussagen entfallen:

I: Und in welcher Sprache konntest du der Geschichte besser folgen?

B: Eher im Deutschen, weil im Französisch haben wir eben ein paar Wörter, die vorkamen, noch nicht so gehabt, und ... ja, (dann?) ist es auch schwerer gefallen, das zu verstehen. (Interview S ANDIV107 CH Z6: 196-197)

Im Gegensatz zu den 40 Lernenden, die MeVoL aufgrund des positiven Lernertrags für die Fremdsprache schätzten, äußerten sich acht Lernende genau gegenteilig. Rückmeldungen wie „,[weil] ich es unnötig und vor allem unnützlich finde (stattdessen können wir Englisch lernen - nur noch 1 Jahr bis zur Prüfung!!!!)“ (Feedbackbogen S NIERRA12 D Z7) oder „[weil] es langweilig ist, nichts bringt und unsere Stunden nutzlos verschwendet" (Feedbackbogen S YVASFE12 Ö Z7) stehen stellvertretend hierfür.

Lediglich vier der ablehnenden Äußerungen bezogen sich auf das didaktischmethodische Design von MeVoL. Dabei wurde je einmal die inhaltliche Wiederholung nach dem deutschen Vorlesepart in der Fremdsprache sowie die Tatsache kritisiert, dass man den Text nicht zweimal hören konnte (Feedbackbogen S LUAMNO02 D Z7). Zwei Äußerungen sprachen sich gegen die Verwendung des Französischen aus.

\subsubsection{Die Wahrnehmung von MeVoL in Abhängigkeit von der verwendeten Fremdsprache (Englisch vs. Französisch)}

Das didaktisch-methodische Design MeVoL wurde in zwei Sprachvarianten umgesetzt: In Deutschland und Österreich wurde als Fremdsprache Englisch verwendet, in der Schweiz hingegen Französisch, da dies eine der offiziellen Landessprachen ist. Bei der

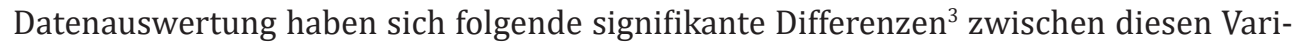
anten in der Akzeptanz des Unterrichtdesigns bei den Schüler/innen gezeigt:

- Fremdsprachliches Niveau: Während für Deutsch-Englisch das Niveau der fremdsprachigen Vorleseteile auf einer sechsstufigen Skala von 80.4\% der Schüler/innen als "genau richtig“ beurteilt wurde $(\mathrm{M}=3.0, \mathrm{SD}=.59, \mathrm{~N}=143)$, wurden die franzö-

3 Sämtliche Unterschiede sind statistisch signifikant (U-Test nach Mann-Whitney, 95\%-Signifikanzniveau). Aus Gründen der besseren Lesbarkeit wird auf die detaillierten teststatistischen Angaben verzichtet. 
sischen Textteile trotz sprachlicher Vereinfachung nur von 55.0\% der Schweizer Schüler/innen als „genau richtig“, von weiteren $42.5 \%$ jedoch als „zu schwer" eingeschätzt $(\mathrm{M}=2.6, \mathrm{SD}=.63, \mathrm{~N}=40)$. Die französische Variante wird, trotz sprachlicher Vereinfachungen, die in den Entwicklungszyklen 6 und 7 umgesetzt wurden, von den Lernenden als bedeutend schwieriger wahrgenommen.

- Verständniserleichterung: In Kombination mit Französisch nehmen die Schüler/innen die Unterstützung des Verständnisses durch die schulsprachlichen Passagen hingegen deutlicher wahr als in der Variante mit Englisch. Für Französisch antworten über drei Viertel (76.6\%) mit „stimmt genau“ (M=3.7, SD=.55, N=43), für Englisch sind es hingegen nur 39.6\% (M=3.1, SD=.84, N=144). Es scheint, dass je schwerer verständlich den Lernenden die fremdsprachigen Textpassagen erschienen, desto bedeutsamer erachteten sie die deutschen Passagen für ihr Verständnis.

- Sequenzierung: Die Mehrheit der Schüler/innen (63.6\%) bezeichnet die Länge der englischsprachigen Sequenzen als "genau richtig“ ( $M=3.0, S D=.80, N=143)$. Bei den französischen Sequenzen schätzen nur 48.8\% die Länge als „genau richtig“ ein, weitere $46.6 \%$ bezeichnen sie jedoch als zu lang bzw. viel zu lang ( $M=3.5, S D=.75$, $\mathrm{N}=41$ ) - und dies trotz der vorgenommenen Anpassung der Sequenzierung, die im französischen Vorlesetext durchschnittlich etwa 200 Wörter im Vergleich zu 400 Wörtern im englischen Vorlesetext umfassen (vgl. 4.1.2).

- Vorlesetexte: Ebenso wurde der französische Vorlesetext im Vergleich zum englischen Text von den Lernenden deutlich negativer wahrgenommen. Die meisten Schüler/innen (44.2\%) gaben an, die französische Geschichte Le jour „eher nicht" interessant zu finden (M=2.6, SD=.72, N=43). Für den englischen Text Paranoid Park liegt der Modus der Antworten mit 46.5\% auf „stimmt genau“, und der Mittelwert ist deutlich höher (M=3.2, $\mathrm{SD}=.96, \mathrm{~N}=144)$.

- Intrinsischer Wert: Das Vorlesen der französischen Textteile ist für die Schüler/innen mit deutlich negativeren Emotionen verbunden als bei den englischen Textteilen. 86.0\% antworten ablehnend mit „stimmt gar nicht“ oder „stimmt eher nicht" auf die Aussage, dass das Vorlesen der französischen Textteile durch die Lehrperson großen Spaß macht ( $M=1.8, \mathrm{SD}=.81, \mathrm{~N}=43)$. Bei den englischen Textteilen liegt die Mehrheit (66.9\%) der Antworten hingegen auf den zustimmenden Antwortkategorien ( $\mathrm{M}=2.7, \mathrm{SD}=.76, \mathrm{~N}=142)$.

- Nutzen und weitere Nutzungsabsicht: Bei der französischen Variante sind deutlich weniger Schüler/innen davon überzeugt, dass MeVoL ihnen im Fremdsprachenunterricht viel gebracht habe als bei der Variante mit Englisch. Die meisten Schweizer Schüler/innen (58.1\%) antworten auf diese Frage mit „stimmt eher nicht“ (M=2.4, $\mathrm{SD}=.54, \mathrm{~N}=43$ ), während die große Mehrheit (81.3\%) der Lernenden aus Deutschland und Österreich mit „stimmt eher" antwortet (M=2.7, SD=.92, N=144). Dementsprechend ist auch die weitere Nutzungsabsicht, die sich in der Haltung ausdrückt, dass MeVoL häufiger im Fremdsprachenunterricht eingesetzt werden sollte, in der Schweiz zurückhaltender (M=2.7, SD=.73, N=43) als in Deutschland und Österreich $(\mathrm{M}=3.0, \mathrm{SD}=1.00, \mathrm{~N}=143)$. 
- Gesamturteil der Schüler/innen: MeVoL hat in Kombination mit Englisch als Fremdsprache den Lernenden deutlich besser gefallen als mit Französisch. Während bei der englischen Variante 39.9\% auf der positivsten Ausprägung antworteten ( $M=3.2$, $\mathrm{SD}=.85, \mathrm{~N}=143$ ), sind es für die französische Variante nur $14.0 \%$, die völlig überzeugt von MeVoL waren (M=2.9, SD=.68, N=43). Über zwei Drittel (67.4\%) antworteten jedoch mit „stimmt eher“.

Diese deutlichen Differenzen der Schülerwahrnehmung von MeVoL in Abhängigkeit von der verwendeten Fremdsprache sind unerwartet und verlangen nach einer Erklärung, die abschließend diskutiert wird (vgl. 5.3).

\subsection{MeVoL aus Lehrersicht}

\subsubsection{Welchen Nutzen sehen die Lehrpersonen in MeVoL?}

Die sieben befragten Kooperationslehrpersonen schätzen den Nutzen von MeVoL insgesamt sehr positiv ein. Die Items in der Dimension „erwarteter Nutzen“ (vgl. Anhang 1) finden zu insgesamt 92.8\% Zustimmung. Am kritischsten beurteilt wird die Interessantheit des französisch-deutschen Vorlesetextes Le jour, die von beiden Schweizer Lehrpersonen als „mäßig“ eingestuft wird. Ebenfalls vergleichsweise wenig Zustimmung erhält die Aussage, dass MeVoL den Genuss bzw. die Freude an Literatur fördert - auch dieser Aussage stimmen die zwei Schweizer Lehrpersonen „eher nicht“ zu. Es ist davon auszugehen, dass auch diese Einschätzung unmittelbar auf den Inhalt des Vorlesetextes zurückzuführen ist. Als nahezu durchgängig sehr nützlich bewertet wird die Kombination von Schul- und Fremdsprache in den Vorlesetexten: So stimmen alle Lehrpersonen der Aussage zu, dass die deutschen Passagen das Verständnis erleichtert haben, sechs Lehrpersonen geben darüber hinaus an, dass die Kombination der beiden Sprachen nicht verwirrend war. Ebenfalls sehr positiv bewertet wird die verständliche und attraktive Aufbereitung der Vorlesetexte. Als Gründe für den Mehrwert von MeVoL nennen die Lehrpersonen:

- die Abwechslung zum normalen Unterricht und die Ergänzung durch das Vorlesen,

- die Erfolgserlebnisse der Schüler/innen, durch die sie sich zutrauen, die Fremdsprache zu verstehen,

- die Auseinandersetzung mit Strategien sowie mit der bewussten Wahrnehmung unterschiedlicher Arten von Kommunikation (Körpersprache, Mimik, Visualisierungen ...),

- die Überzeugung, dass einige Lernende durch MeVoL ihre Fremdsprachenkenntnisse verbessern konnten,

- die Möglichkeit, mithilfe von MeVoL Jugendlichen Literatur nahezubringen. 
Besonders positiv hervorgehoben wird die Andersartigkeit von MeVoL gegenüber anderen eingesetzten Methoden im Unterricht:

Ja, dass sie eben mal einen ganz anderen Zugang haben zu dem Fach. Dass sich langsame oder schwächere Schüler als kompetent erleben. Das finde ich einen Mehrwert. Und ja, dass das als Genuss erlebt wird und auch mal entspannt. Ich glaube, viele Schüler sind oft immer sehr angespannt im Unterricht, und man fordert ja auch viel von denen. Und es ist viel drum herum an Stress an so einem Vormittag oder in so einer Woche und dann ist das vielleicht in der Zeit auch so wie so eine Insel, wo viele Schüler sich darauf freuen. (Interview LP 1 D Z6: 87-88)

Gerade in den Klassen, in denen deutsch-französisch vorgelesen wurde, sehen die Lehrpersonen in MeVoL auch einen Motivationsfaktor für das Fach Französisch an sich:

Wenn ich jetzt im Französisch die Motivation messen könnte, wäre sie jetzt definitiv höher als vorher. Ganz sicher. Ganz klar. Weil es ist wirklich ein Herauslösen aus dem Alltagstrott. Es ist das Spezielle, das Außergewöhnliche, das Offizielle. All diese Sachen machen solche Unterrichtsreihen schon sehr attraktiv. (Interview LP 3 CH Z6: 136-141)

\subsubsection{Mit welchem Aufwand ist MeVoL für die Lehrpersonen verbunden?}

Der Aufwand, der den Lehrpersonen durch MeVoL erwächst und der im Akzeptanzmodell (vgl. 2.2.2) dem Nutzen gegenübersteht, wird von den Lehrpersonen durchgehend als „eher angemessen“ oder „völlig angemessen“ eingestuft. Aus den Interviews geht allerdings hervor, dass der von den Lehrpersonen wahrgenommene Vorbereitungsaufwand stark variiert. Eine Lehrperson berichtet von einer intensiven Auseinandersetzung mit dem Text, während eine andere für die flexible Ausgestaltung der Vorlesesequenz plädiert:

Ich hatte nach dem ersten Vorlesen gemerkt, dass ich mich noch intensiver mit dem Text auseinandersetzen muss. Einfach, dass ich selber mehr Sicherheit habe, dass ich selber mehr spielen kann. [...] Im weiteren Verlauf habe ich dann gemerkt, dass es wirklich wichtig ist, [...] dass mich der Text begleitet über einige Tage, einfach dass ich besser reinkomme, dass es mehr mein Text wird, irgendwie. (Interview LP 3 CH Z6: 25 und 33)

Ich denke, dass ich das so ja spontaner und auch ohne extreme Vorbereitung ganz gut hinkriege, und deswegen habe ich mir angeschaut, was ist organisatorisch zu tun, was für Impulse kann ich noch geben, wo ist was, wo ich mehr machen will, wo ist weniger. (Interview LP 1 D Z6: 33-34)

Am kritischsten beurteilt wird der Aufwand, der durch den Einsatz von MeVoL im Unterricht grundsätzlich entsteht. Für eine Lehrperson ist der Aufwand von der Anzahl der zur Verfügung stehenden Wochenstunden abhängig. 


\subsubsection{Wie schätzen die Lehrpersonen das Interesse anderer Lehrpersonen und die Rahmenbedingungen für die Umsetzung von MeVoL in ihrem Unterricht ein?}

Als soziale Einflussfaktoren für die Nutzungsabsicht gelten zum einen die Absicht von Kolleg/innen, MeVoL zu nutzen, zum anderen die Kompatibilität mit den eigenen Erwartungen an guten Sprachunterricht. Ob das Design tatsächlich zum Einsatz kommt, wird letztlich auch wesentlich von den schulischen Rahmenbedingungen beeinflusst.

Bezüglich der sozialen Einflussfaktoren zeigt sich in den Antworten und Einschätzungen der Lehrpersonen ein uneinheitliches Bild. Vier Lehrpersonen geben an, dass andere Kolleg/innen Interesse an MeVoL bekundet haben:

Und es ist ja auch ein Thema im Schulhaus. Also es sind jetzt auch immer wieder Lehrer auf mich zugekommen, und ich werde das Projekt dann schon noch vorstellen in der nächsten Oberstufensitzung, was genau im Detail lief. Aber es kamen jetzt schon sehr viele nachhaken. Was das sei, und ... spannend, und erzähl mal. (Interview LP 3 CH Z6: 117)

Drei Lehrpersonen berichten hingegen, dass ihr Kollegium nicht explizit Interesse am Projekt zeigte. Gleichzeitig gibt der überwiegende Teil der Lehrpersonen an, dass eine interne Fortbildung an der Schule „eher“ auf Interesse stoßen würde.

Als potenziell negativer sozialer Einflussfaktor wird die z.T. kritische Haltung des Kollegenkreises gegenüber zeitaufwändigen Aktivitäten genannt:

[...] Der Zeitdruck war jedoch groß. Und diese Zeit mit der Klasse, zusätzliche Dinge zu machen, die Zeit benötigen, das [...] verursacht immer einen Druck, ja. Es war auch zu wenig Zeit, um sich mit dem [...] gesamten Klassenteam abzustimmen. Und wenn Dinge passieren, die nicht vollkommen transparent sind, ist oft ein gewisser Unmut auch da. Das ist ganz automatisch, aber trotzdem ist es sehr umfangreich, Kollegen auf so ein Projekt einzuschwören oder auch zu informieren, weil's so umfangreich ist und weil die Hintergründe auch transportiert werden müssen. Und die Haltung ist meistens so: „Ich möchte zwar alles mitbekommen, aber bitte lasst mich mit Arbeit und mit Zusatzinformation in Ruhe.“ Das ist so eine diffizile Sache, oder? (Interview LP 2 Ö Z6: 38-39)

Große Zustimmung findet die Aussage, dass sich MeVoL gut in den eigenen Fremdsprachenunterricht einbinden lässt. Dasselbe gilt für einzelne Komponenten des Designs, konkret für das scaffolding, die Anschlusskommunikation sowie für das Strategietraining. Etwas kritischer, aber überwiegend positiv wird auch die Anschlussfähigkeit von $\mathrm{MeVoL}$ an den restlichen Unterricht beurteilt. Insbesondere in den Interviews werden allerdings häufig die beschränkten zeitlichen Ressourcen im schulischen Alltag als Schwierigkeit bei der Umsetzung von MeVoL genannt. Als Argument für eine zeitliche Beschränkung der Vorlesetage wird auch die Verteilung der einzelnen Unterrichtseinheiten auf die Wochentage angeführt:

[...] Und ich wollte es einfach auch möglichst bündig lesen, man vergisst Dinge wieder, wenn man zu lange Pausen drin hat, [...] es ist eigentlich besser, man hat es am Stück, 
und die wollten das auch, fanden es ja spannend und wollten auch, dass man vorankommt. (Interview LP1 D Z6: 27-28)

\subsubsection{Haben die Lehrpersonen die Absicht, MeVoL weiterhin einzusetzen?}

Unter den MeVoL-Kooperationslehrpersonen ist die Absicht, das Unterrichtsdesign auch weiterhin einzusetzen, durchgängig gegeben (5 „völlig“, 2 „eher“). Nahezu alle beabsichtigen auch, MeVoL anderen Kolleg/innen weiterzuempfehlen, wobei eine Kollegin aufgrund der wenigen konkreten Aufgaben für die Schüler/innen davon eher absehen wird. Als Gründe für eine mögliche Weiterempfehlung wird zum einen die fächerübergreifende Anlage des Designs erwähnt: „[...] Also das muss ein Element werden von einem guten Sprachunterricht. Man spricht ja immer davon, dass man fächerübergreifend arbeiten sollte und Vernetzungen herstellen. Unbedingt, unbedingt" (Interview LP 3 CH Z6: 264-267). Zum anderen wird auch das von der Lehrperson wahrgenommene Kompetenzerleben der Lernenden genannt:

Es ist etwas, das bei uns in unseren Büchern, Lehrbüchern, Lernbüchern eigentlich so nicht vorkommt. Wir haben so dieses Klassische, ganze Texte, einfache Texte in Englisch und „Speak about it“ und so weiter. Und diese Herangehensweise hat bei manchen, eher bei schwächeren Kindern, Kindern, die nicht gerne lesen, immer so das Problem. Ja, Überforderung, sie stellen sehr schnell ab, weil einfach zu viel - auch wenn es ein relativ kurzer Text ist, aber für sie individuell empfunden zu viel englische Texte da sind. [...] Und mir hat ganz am Schluss, von der, von unserem Projekt eine, die schwächste Schülerin, die ich habe, also eine Integrationsschülerin, die kam und hat gesagt: „Herr Lehrer, warum hören wir dann wieder auf? Das hat wirklich Spaß gemacht. Ich habe Englisch verstanden.“ Und ich bin sicher, auch wenn sie zwei Drittel nur deshalb verstanden hat, weil Deutsch dabei war, aber völlig egal. Das Empfinden war: „Ich verstehe Englisch.“ (Interview LP 3 Ö Z6: 124)

\subsection{Zusammenfassung}

Die Akzeptanz von MeVoL aus der Perspektive von Lehrpersonen und Schüler/innen, die in den letzten Abschnitten anhand verschiedener Datenquellen im Detail dargestellt wurde, wird nun in einer synoptischen Übersicht zusammengefasst (vgl. Tab. 2). Sie orientiert sich an den Dimensionen des verwendeten Akzeptanzmodells sowie des Erwartungs-Wert-Modells der Motivation (vgl. 2.2) und differenziert zwischen Bereichen, in denen die Akzeptanz als erfüllt betrachtet werden kann, und solchen, in denen die Akzeptanz kritisch einzustufen ist. 
Tabelle 2: Synoptische Darstellung der Akzeptanz von MeVoL aus Lehrer- und Schülersicht

\section{Akzeptanz vorhanden}

\begin{tabular}{|c|c|}
\hline Lehrpersonen & Schüler/innen \\
\hline $\begin{array}{l}\text { Nutzen: } \\
\text { - Kombination von Schul- und Fremdsprache } \\
\text { sinnvoll } \\
\text { - Neuigkeit von MeVoL als gute Abwechslung } \\
\text { bzw. Ergänzung zum normalen Unterricht } \\
\text { - gesteigertes Kompetenzerleben der S fest- } \\
\text { stellbar } \\
\text { - Auseinandersetzung mit Rezeptionsstrategi- } \\
\text { en sinnvoll } \\
\text { - Steigerung der Fremdsprachenkenntnisse der } \\
\text { S feststellbar } \\
\text { Aufwand: } \\
\text { - angemessener Arbeitsaufwand für LP, jedoch } \\
\text { individuell stark unterschiedlich } \\
\text { - verständliche und attraktive Aufbereitung der } \\
\text { Vorlesetexte und Aufgaben vereinfacht Ein- } \\
\text { satz im Unterricht } \\
\text { Rahmenbedingungen: } \\
\text { - Integration in eigenen Fremdsprachenunter- } \\
\text { richt gut möglich } \\
\text { Nutzungsabsicht: } \\
\text { - weitere Nutzungsabsicht bei allen LP vorhan- } \\
\text { den } \\
\text { - nahezu alle LP wollen MeVoL Kolleg/innen } \\
\text { weiterempfehlen }\end{array}$ & $\begin{array}{l}\text { Intrinsischer Wert: } \\
\text { - MeVoL macht Spaß, ist interessant und gefällt } \\
\text { den Lernenden } \\
\text { - gute Abwechslung zum regulären Unterricht } \\
\text { - Abschalten/Entspannen durch rezeptiven Mo- } \\
\text { dus möglich } \\
\text { - positive Bewertung/Interessantheit der Texte } \\
\text { - positivere Emotion und Motivation im Ver- } \\
\text { gleich zum bisherigen Unterricht (affective } \\
\text { value) } \\
\text { - Kompetenzerleben durch verbessertes Text- } \\
\text { verständnis aufgrund der Sprachkombination } \\
\text { - MeVoL wird gegenüber anderen Formen der } \\
\text { Textrezeption (Lesen nur in der FS, lautes Vor- } \\
\text { lesen durch S, Texte auf CD hören, stilles Le- } \\
\text { sen) präferiert } \\
\text { Nutzen: } \\
\text { - Kombination von Schul- und Fremdsprache } \\
\text { sinnvoll } \\
\text { - positiver Lerngewinn (cognitive value) für } \\
\text { Fremdsprache im Allgemeinen (Wortschatz, } \\
\text { Aussprache, Hörstrategien) } \\
\text { - deutsche Textteile, Sprechen über Geschichte } \\
\text { sowie Bilder sind hilfreich für das Verständnis } \\
\text { - bessere Organisation im Vergleich zum bishe- } \\
\text { rigen Unterricht } \\
\text { Aufwand: } \\
\text { - geringerer erlebter Aufwand im Vergleich zum } \\
\text { bisherigen Unterricht } \\
\text { - Vorlesen der Lehrperson ist im Vergleich zum } \\
\text { Selber-Lesen entlastend } \\
\text { Nutzungsabsicht: } \\
\text { - } 76 \% \text { der S möchten nochmals eine mehrspra- } \\
\text { chige Geschichte vorgelesen bekommen }\end{array}$ \\
\hline
\end{tabular}

$S=$ Schüler, $L P=$ Lehrperson, $F S=$ Fremdsprache 
Akzeptanz kritisch

\begin{tabular}{|c|c|}
\hline Lehrpersonen & Schüler/innen \\
\hline $\begin{array}{l}\text { intrinsischer Wert: } \\
\text { - Interessantheit des französischen Vorlesetex- } \\
\text { tes nur mäßig } \\
\text { Aufwand und Rahmenbedingungen: } \\
\text { - Einsatz von MeVoL im Unterricht wird gefähr- } \\
\text { det durch zeitlich beschränkte Ressourcen } \\
\text { und Konkurrenz mit anderen Unterrichtsinhal- } \\
\text { ten } \\
\text { Soziale Einflussfaktoren: } \\
\text { - moderates Interesse weiterer LP an MeVoL } \\
\text { - moderates Interesse an internen Fortbildun- } \\
\text { gen zu MeVoL an den Schulen } \\
\text { - kritische Haltung von Kolleg/innen gegenüber } \\
\text { zusätzlichen, zeitaufwändigen Aktivitäten }\end{array}$ & $\begin{array}{l}\text { intrinsischer Wert: } \\
\text { - Langeweile/keine Abwechslung in MeVoL } \\
\text { - negative Bewertung der Vorlesetexte (v.a. } \\
\text { französischer Text) } \\
\text { Aufwand: } \\
\text { - Verständnisschwierigkeiten (v.a. auf Franzö- } \\
\text { sisch) } \\
\text { - Niveau der französischen Textteile zu schwer } \\
\text { - französische Sequenzen zu lang } \\
\text { Nutzen: } \\
\text { - Sinn der Anschlusskommunikation wird nicht } \\
\text { verstanden } \\
\text { - geringerer wahrgenommener Nutzen der Be- } \\
\text { schäftigung mit Hörstrategien und Aufgaben } \\
\text { nach dem Vorlesen } \\
\text { - Lerngewinn (cognitive value) wird vergleich- } \\
\text { bar mit bisherigem Unterricht oder negativer } \\
\text { eingeschätzt (v.a. bei Französisch) } \\
\text { - Kritik am Design (inhaltliche Wiederholung } \\
\text { nach deutschem Vorleseteil, keine Möglich- } \\
\text { keit, den Text ein zweites Mal zu hören) } \\
\text { - Verwendung von Französisch als Fremdspra- } \\
\text { che wird grundsätzlich kritisiert } \\
\text { Nutzungsabsicht: } \\
\text { - geringere Nutzungsabsicht bei Französisch } \\
\text { als bei Englisch }\end{array}$ \\
\hline
\end{tabular}

$L P=$ Lehrperson

Fasst man die Ergebnisse auf einer höheren Abstraktionsebene zusammen, so kann die Akzeptanz des mehrsprachigen Unterrichtsdesigns MeVoL insgesamt sowohl bei den Lehrpersonen als auch bei den Schülerinnen und Schülern als vorhanden angesehen werden. Beide Personengruppen beurteilen den Nutzen insgesamt positiv, der Aufwand wird hauptsächlich von den Lernenden bei gleichem Lerngewinn als geringer als im herkömmlichen Unterricht eingestuft. Sie schätzen es, durch das Zuhören im rezeptiven Modus entlastet zu sein und entspannen zu können. MeVoL überzeugt die Lernenden vor allem auch aufgrund seines intrinsischen Wertes: Das Hören von interessanten Geschichten macht ihnen zum Großteil Spaß und ist damit mit positiven Emotionen (affective value) und gesteigerter Motivation verbunden; durch die Sprachkombination können sie mehr verstehen als bei ausschließlich fremdsprachigen Texten, was zu einem gesteigerten Kompetenzerleben beiträgt. MeVoL wird deshalb anderen Formen des (Vor-)Lesens im Fremdsprachenunterricht vorgezogen. Sowohl Lehrpersonen als auch Lernende sehen in der Neuartigkeit des Unterrichtsdesigns einen Mehrwert, der als gute Abwechslung bzw. Ergänzung zum herkömmlichen Fremdsprachenunterricht angesehen wird. Die innovative Grundidee des Unterrichtsdesigns, namentlich die Kombination von Schul- und Fremdsprache in einem Vorlesedesign auf der Sekundar- 
stufe I, findet damit breite Akzeptanz sowohl bei den Lernenden als auch bei den Lehrenden. Die Sinnhaftigkeit und Nützlichkeit des Designs als Ganzes ist für die Akteur/ innen vorhanden.

Abschließend sollen nun jedoch jene Bereiche diskutiert werden, in denen die Akzeptanz von MeVoL eher kritisch ausfällt. Diese Bereiche bedürfen besonderer Beachtung, da sie für die weitere Verwendung des Unterrichtsdesigns MeVoL besonders relevant sind. Sie stellen die noch vorhandenen „Ecken und Kanten“ des Designs dar, die weiterer Anpassungen bzw. der genaueren Untersuchung bedürfen:

Geringere Akzeptanz von MeVoL in Kombination mit Französisch: MeVoL wird in Kombination mit Französisch als Fremdsprache im Vergleich zu Englisch von Lehrpersonen und Schüler/innen deutlich weniger akzeptiert: Der französische Vorlesetext wurde als weniger interessant beurteilt, die französischen Textteile wurden trotz Anpassungen als zu lang und als zu schwierig eingestuft, weshalb die Lernenden deutlich mehr über Verständnisschwierigkeiten klagten, und der Lerngewinn von MeVoL wurde deutlich geringer beurteilt. Wir vermuten, dass mehrere Gründe dafür verantwortlich sind: Erstens ist die grundsätzliche Einstellung der Schüler/innen zu Französisch als Fremdsprache kritischer als zu Englisch (8 negative Codings für Französisch gegenüber 5 positiven Codings für Englisch in 18 Schülerinterviews). Damit ist auch die Motivation, sich mit französischsprachigen Texten zu beschäftigen, geringer als bei englischsprachigen Texten:

I: Wie geht es dir dabei, wenn du eine Geschichte oder einen Text auf Französisch liest?

B: Ja, also ... also Französisch ist nicht so meine Lieblingssprache. Und, ja, also [...] ich finde es nicht so cool, weil Französisch habe ich eigentlich nicht so gern. [...]

I: Mhmm. Wie fühlst du dich denn, wenn du etwas lesen musst im Französisch?

B: Ja, eigentlich gut, einfach ... es ist dann einfach beim Laut-Vorlesen, dann ist ... dann habe ich einfach jeweils Angst, z.B. wenn ich etwas falsch sage oder so, also ein Wort, welches ich falsch ausspreche oder so. Aber sonst eigentlich gut.

I: Mhmm. Ist es im Englisch anders?

B: Ja, Englisch habe ich ein wenig lieber, und dort ... lese ich auch lieber Sachen, wie im Französisch.

I: Weißt du warum?

B: Ich weiß auch nicht, Französisch ist so ... irgendwie schwieriger und so, und Englisch ist halt einfach ein wenig einfacher und ist auch, also auch wichtiger, weil das kann man eigentlich überall reden, [...] ja. (Interview S LEERDI05 CH: 36-47)

Ein weiterer Grund ist, dass das sprachliche Niveau der Schüler/innen in Französisch aufgrund der geringeren Anzahl Lernjahre (vgl. Tab. 2 in 2.1) sowie der geringeren Re- 
levanz im Alltag der Jugendlichen tatsächlich geringer ist als in Englisch. ${ }^{4}$ Die Lernenden scheinen zudem in Bezug auf Englisch mehr Selbstvertrauen in ihre sprachlichen Fähigkeiten zu haben als bei Französisch, wie obiges Beispiel ebenfalls zeigt.

Gelingende Anschlusskommunikation: Eine lebendige Anschlusskommunikation fand in den beobachteten Unterrichtsstunden kaum statt. Wir machen dafür verantwortlich, dass das bewertende Sprechen über Texte für viele Schüler/innen ungewohnt ist. Es gehört nicht zu ihren schulischen Handlungsroutinen und löst daher Irritation bzw. Unsicherheit aus. Das Schaffen eines Settings, das eine aktive persönliche literarische Auseinandersetzung im Schulkontext ermöglicht, ist eine große Herausforderung. Auch scheinen die Lehrpersonen damit mehrheitlich nicht vertraut zu sein und bedürfen wahrscheinlich stärkerer methodischer Anleitung. Zentral ist dabei, dass den Lernenden der Sinn der Anschlusskommunikation erklärt wird und dass Hemmungen bei den Lernenden abgebaut werden, sich persönlich zu einem Text zu äußern. Das Anerkennen, dass es verschiedene Deutungen eines Textes gibt und dass die Auseinandersetzung mit Meinungen anderer Personen durchaus interessant sein kann, sind dafür wichtige Voraussetzungen. Aus mehrsprachigkeitsdidaktischer Perspektive hat die Anschlusskommunikation nicht zwingend in der Fremdsprache zu erfolgen, sondern kann auch in der Schulsprache stattfinden. Dadurch kann zumindest eine sprachliche Hürde bei den Lernenden abgebaut werden, sich zum Text zu äußern.

Vermittlung und Festigung von Hörstrategien: Der Nutzen der Hörstrategien für das Verständnis wurde von den Lernenden nur als moderat wahrgenommen, obwohl die Lehrpersonen die Vermittlung von Hörstrategien als sehr sinnvoll beurteilt haben. Wir vermuten daher, dass die Thematisierung und Reflexion von Hörstrategien in MeVoL noch optimiert werden kann. Folgende Punkte sollten dabei berücksichtigt werden: Erstens müssen Lehrpersonen den Einsatz von Hörstrategien modellieren, bis diese selbstständig von den Lernenden angewendet werden. Ein schrittweiser und wiederholter Aufbau von Strategien durch Modellierung ist dabei wichtig. Zweitens reicht es nicht aus, die verwendeten Hörstrategien von den Lernenden mehrmals mit demselben Instrument erfassen zu lassen. Die Schüler/innen sollen gezielt dazu angehalten werden, ihren Hörstrategieeinsatz zu reflektieren und zu optimieren, indem sie neue Strategien ausprobieren und damit ihr Repertoire an sinnvollen Strategien erweitern. Dazu ist es wichtig, dass Lernende die positive Erfahrung machen, dass ihnen der Einsatz neuer Strategien einen Gewinn verschafft und daher nützlich für sie ist. Drittens benötigen der Aufbau und die Festigung von Hörstrategien viel Zeit. Da eine MeVoL-Vorle-

4 Die Evaluation der Fremdsprachkompetenzen im Bildungsraum Zentralschweiz zeigt, dass die Schüler/innen am Ende der 8. Klasse eine deutliche bessere Schreib- und Lesekompetenz in der ersten Schulfremdsprache Englisch haben als in Französisch (vgl. Peyer et al. 2016: 48f.). Beim Hörverstehen in Französisch erreicht nur eine Minderheit von 8.7\% der Lernenden am Ende der 8. Klasse das Lehrplanziel für die obligatorische Schulzeit. Etwa ein Viertel (28.7\%) hat zu diesem Zeitpunkt selbst das Lehrplanziel für die 6. Klasse noch nicht erreicht (vgl. ebd.: 39). 
sesequenz von vergleichsweise kurzer Dauer ist, ist es empfehlenswert, Hörstrategien auch nach dem Einsatz von MeVoL weiterhin im Unterricht zu thematisieren und zu reflektieren. Eine Checkliste mit einer Sammlung von Hörstrategien, die die Schüler/ innen erhalten, kann dafür hilfreich sein.

Konkurrenz von MeVoL mit anderen Anforderungen an Lehrpersonen: Die Ergebnisse der Datenauswertung zeigen, dass die Akzeptanz von MeVoL bei den Lehrpersonen grundsätzlich gegeben ist, in der praktischen Umsetzung MeVoL jedoch in Konkurrenz mit den schulischen Rahmenbedingungen und Anforderungen an die Lehrpersonen steht. Hierbei handelt es sich allerdings um eine Problematik, die nicht spezifisch für MeVoL ist, sondern für jegliche schulische Innovation besteht, die mit einem gesteigerten Aufwand für die Lehrpersonen verbunden ist. Lehrpersonen stehen unter Druck, die Inhalte des Lehrmittels bzw. Lehrplans umzusetzen, und sind mit vielen Erwartungen und Aufgaben konfrontiert, mit denen MeVoL konkurriert:

[...] Es gab viele, die neugierig waren, und auch einfach eben weil sie dich im Hause sahen oder dich kennen, und dann fragen sie nach und so. Aber ich denke einfach diese Frage, die auf dem Interviewblatt war, [Weiterbildung] fürs ganze Team, ist bei uns nicht aktuell, weil bei uns einfach dermaßen viel sonst läuft, oder. (Interview LP ${ }_{5} \mathrm{CH} \mathrm{Z6}_{2}$ 267)

Aus diesem Grund scheint es uns besonders wichtig, dass für die weitere Verbreitung des Unterrichtsdesigns besonders auf die Integrations- und Anschlussfähigkeit von MeVoL im regulären Unterrichtsgeschehen geachtet wird. 